\title{
Synthesis of New Fluorinated Fused
}

\section{Heteropolycyclic Nitrogen Systems Containing a Pyrazolotriazine Moiety as Antimicrobial Agents Part I}

\author{
Layla A. Taib, Sofiyah A. Adibani* \\ Department of Chemistry, Faculty of Science, King Abdul Aziz University, Jeddah, KSA \\ Email: *laylataib@yahoo.com, sanbnsm@hotmail.com
}

How to cite this paper: Taib, L.A. and Adibani, S.A. (2018) Synthesis of New Fluorinated Fused Heteropolycyclic Nitrogen Systems Containing a Pyrazolotriazine Moiety as Antimicrobial Agents Part I. International Journal of Organic Chemistry, 8, 176-189.

https://doi.org/10.4236/ijoc.2018.81013

Received: February 6, 2018

Accepted: March 19, 2018

Published: March 22, 2018

Copyright $\odot 2018$ by authors and Scientific Research Publishing Inc. This work is licensed under the Creative Commons Attribution International License (CC BY 4.0).

http://creativecommons.org/licenses/by/4.0/

\begin{abstract}
A simple route to synthesize novel fluorinated fused hetero-polycyclic nitrogen systems containing a pyrazolotriazine moiety $(5,8,11)$ have been deduced from cyclization of 2-aminothiocarbonyl-5-arylidene-3-phenyl-1,2,4-triazin$6(1 \mathrm{H})$ one $(2)$ with diethoxy-phosphine, diethyl carbonate and/or diethyl oxalate in boil THF followed by cyclo condensation with aryl sulfonic acid hydrazide in EtOH/piperidine and finally fluorination with trifluoroethyl acetate. Structures of the products have been established from their elemental analysis and spectral measurements. The antimicrobial activity of the targets has also been evaluated.
\end{abstract}

\section{Keywords}

Synthesis, Fluoro-Fused Heterocyclic Systems, Antimicrobial Agents

\section{Introduction}

The introduction of phosphorus atom to heterocyclic nitrogen system improves their biocidal properties, which may be the unique behavior of donation and back donation of a phosphorus atom $(\mathrm{P} \sim \mathrm{N})$ [1] [2]. Most of the organic phosphorus systems exhibited high biocidal properties as herbicides, insecticide, and pesticide [3] [4] [5]. 1,2,4-Triazine derivatives are a prominent structural core system present in numerous biologically active compounds, exhibit a wide range of biological activities, as anticancer [6], antitumor [7] [8] [9], anti-inflammatory, anti-fungal, antibacterial [10] [11] [12] [13] [14] as well as potential inhibitors [15]. 
Zrin et al. [6] [7] [8] [9] [10], reported that 2-aminothiocarbonyl-1,2,4-triazin6-ones have a significantly greater cytotoxic effect compared to that other compounds. Functionalized 1,2,4-triazines are used to remove metal ions by formation an stable metal complexes [16]. On the other hand, substituted pyrazoles bearing or containing a 1,2,4-triazine nucleus, showed a wide spectrum of biological activities [17]. A survey of the literature revealed that fluorine atoms bonded with 1,2,4-triazines often enhance their properties [18] [19] [20]. Upon these observations, the present work tends to synthesize of novel fluorine substituted pyrazolotriazine derivatives, and evaluation of their antimicrobial activity.

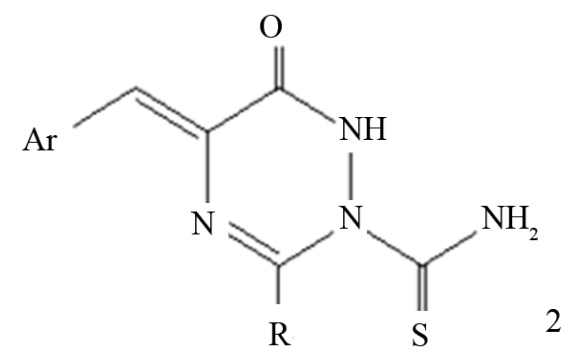

\section{Chemistry}

4-(4'-Chloro-3-phenyl)-oxazol-5-one (1) used as starting material obtained according to the repeated method [9]. The interaction between compound 1 and thiosemicarbazide in refluxed glacial acetic acid yielded 2-aminothiocarbonyl1,2,4-triazine-6 $(1 \mathrm{H})$ one 2 . Ring closure reaction of 2 with diethoxy phosphine under reflux with THF afforded 7-(4'-chlorobenzylidene)-9-phenyl-2-thioxo $[5,1,2,4]$ phospha[1,2,4]triazolo[1,2-a][1,2,4]triazin-6-one (3). The main aim of the present work is to obtain fluorinated fused hetero-polycyclic nitrogen systems containing a pyrazolotriazine moiety. Thus, cyclocondensation of compound 3 with 4-toluenesulffonyl hydrazide in reflux ethanol-piperidin led to the direct formation of pyrazolo[4,3-e][1,2,4]triazino[1,2-a][1,2,4]triazolone 4 , which upon treatment with trifluoroethyl acetate afforded $\mathrm{N}$-(trifluoroacetyl)N'(4-toluylsulfonyl)hydrazine derivative 5 (Scheme 1). Presence of piperidine used as an oxidizing agent for pyrazole moiety. Formation of compound $\mathbf{5}$ from $\mathbf{2}$ as shown in (Figure $1 \&$ Figure 2).

Formation of compound 2 from the oxazolidinone 1 may be tacks place via a double nucleophilic attack of $\mathrm{NH}_{2}$ and $\mathrm{NH}$ to the cyclic ketone followed by carbon-enolic center (Figure 1), while formation of 3 from compound 2 may be a nucleophilic of $\mathrm{NH}_{2}$ and $\mathrm{NH}$ of compound 2 to the P-OR center followed by cyclocondensation with acid hydrazide and finally fluoroacylation (Figure 2).

Similarly, acylation of compound 2 by reflux with diethyl carbonate in a non polar solvent as THF, produced the 2-thioxo-1,2,4-triazolo-1,2,4-triazin-7,9-dione (6). Full hydrocyclization of compound 6 by reflux with 4-toluenesulfonic acid hydrazide in ethanol with drops of piperidine, afforded the fused hetero-polycyclic system 7 via its warming with trifluoroethyl acetate, yielded the 


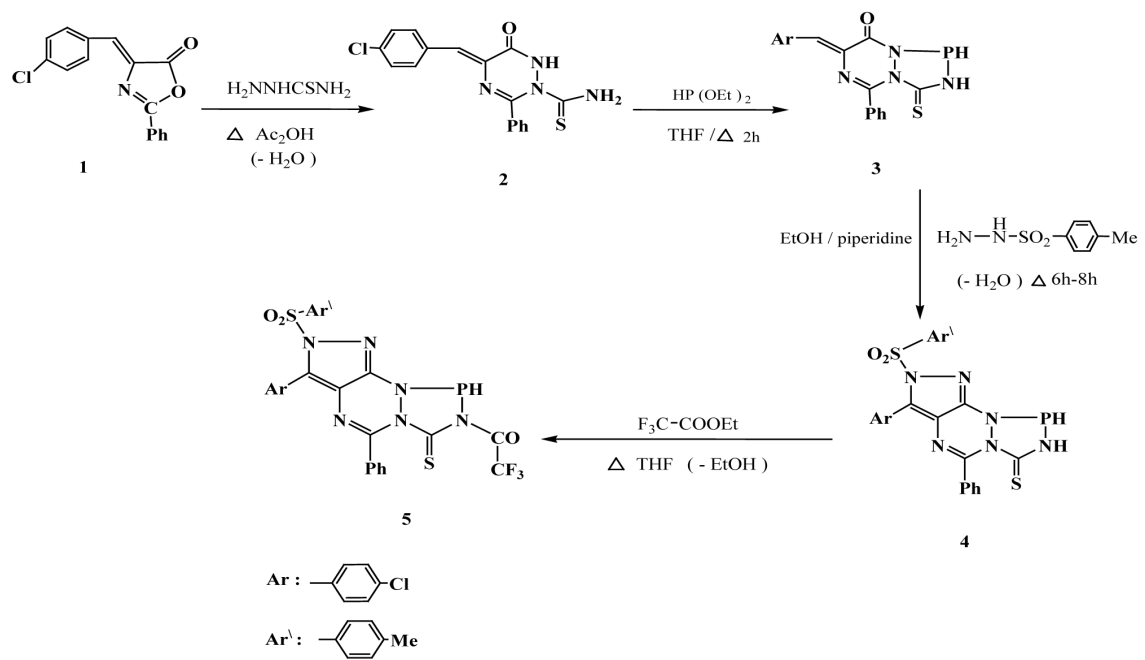

Scheme 1. Formation of 5 from 2.

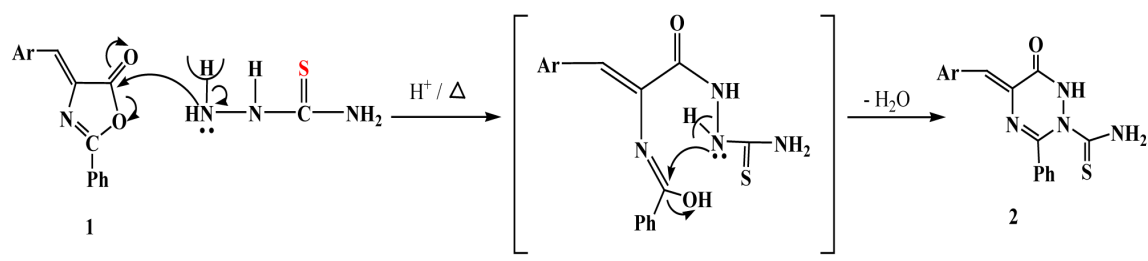

Figure 1. Formation of compound 2 from 1.

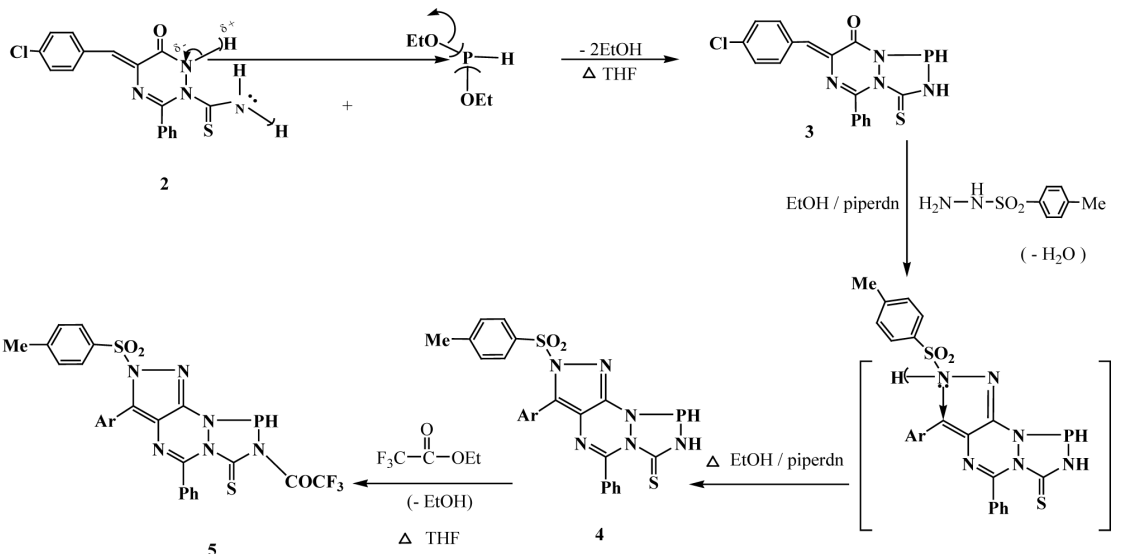

Figure 2. Formation of compound 5 from 2.

corresponding $\mathrm{N}$-trifluoroacetyl derivative 8 (Scheme 2). Formation of compound 8 from 2 may be as shown in (Figure 3).

Due to the biological activity of fluorine-substituted 3-thioxo-1,2,4-triazin-5ones [4] [5], the present work tends to synthesize of 2-(4'-tollylsulfonyl)-3(4'-chlorophenyl)-5-phenyl-7-thioxo-8-trifluoroacetylpyrazolo[4,3-e][1,2,4]triaz ino[1,2-a] [1,2,4]triazin-9,10-dione (11) starting from reflux of compound 2 with diethyl oxalate in THF followed by heterocyclization with sulfonyl hydrazide and finally fluoroacylation via warming with trifluoroethyl acetate in dioxane (Scheme 3). 
<smiles>NC(=S)N1NC(=O)/C(=C/c2ccc(Cl)cc2)N=C1P</smiles><smiles>CCOC(=O)C(F)(F)F</smiles>

8

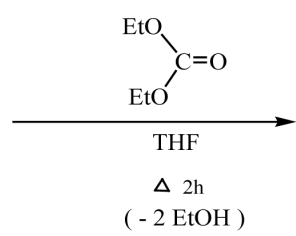<smiles>O=C1C(=C[Tl])N=C(c2ccccc2)n2c(=S)[nH]c(=O)n21</smiles><smiles>Cc1ccc(OS(N)(=O)=O)cc1</smiles>

$(1: 1)$ EtOH / piperidine $\left(-\mathrm{H}_{2} \mathrm{O}\right)$ $\triangle 6 \mathrm{~h}-8 \mathrm{~h}$

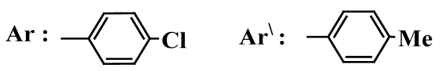

Scheme 2. Formation of 8 from 2.<smiles>NC(=S)N1NC(=O)/C(=C/c2ccc(Cl)cc2)N=C1c1ccccc1</smiles><smiles></smiles>

11

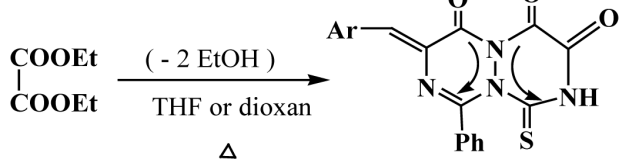

9 $\begin{array}{ll}\mathrm{H}_{2} \mathrm{~N} \cdot \mathrm{N} \cdot \mathrm{SO}_{2}-\mathrm{Me} & \begin{array}{l}\mathrm{EtOH} / \text { piperidine } \\ (1: 1)\end{array} \\ \left(-\mathrm{H}_{2} \mathrm{O}\right)\end{array}$<smiles>O=c1[nH]c(=S)n2c(-c3ccccc3)nc3c([Al])n([Al])nc3n2c1=O</smiles>

10

Ar :

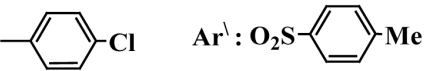

Scheme 3. Formation 11 from 2.

The higher stability of polyheterocyclic systems 3, 4, 6, 7, 9 and 10 may be due to the presence of thion thiol and keto enol forms as tautomeric structures (Figure 4).

\section{Result and Discussion}

The former structure of new compounds 2-11 established from their correct 


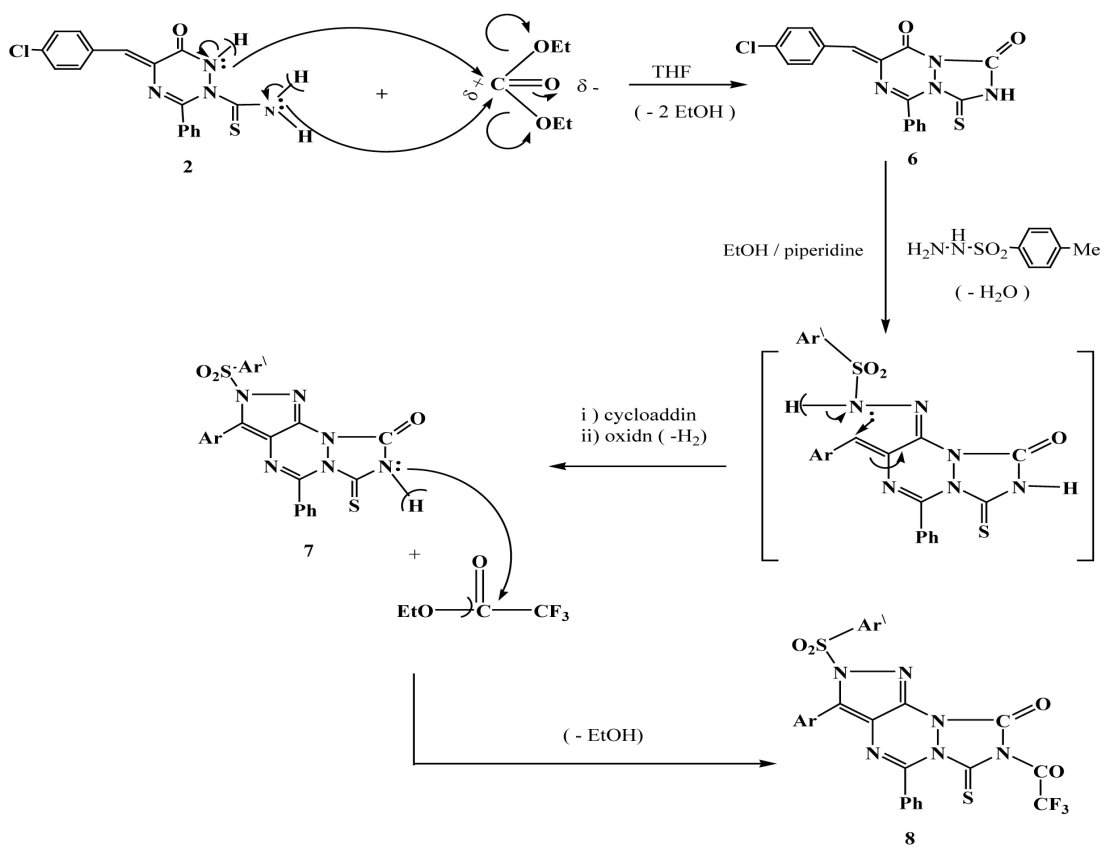

Figure 3. Formation of 8 from 2.
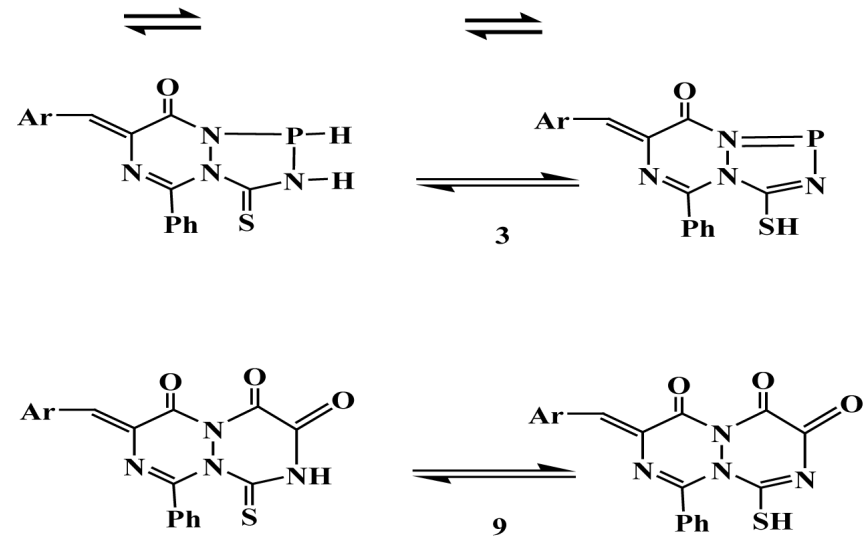

Figure 4. Tautomeric structures.

elemental analysis and spectral measurements. Compound 1 prepared according to the literature reported method [8]. The IR spectrum showed the absorption bands at $\gamma 1700,1610,1596$, and $1070 \mathrm{~cm}^{-1}$ for $\mathrm{C}=\mathrm{O}, \mathrm{C}=\mathrm{N}, \mathrm{C}=\mathrm{C}$ and $\mathrm{C}-\mathrm{O}-\mathrm{C}$ functional groups, while that of compound 2 recorded additional bands at $\gamma$ 3250,3159 and $1210 \mathrm{~cm}^{-1}$ for new $\mathrm{NH}, \mathrm{NH}_{2}$ and $\mathrm{C}=\mathrm{S}$ groups.

The ${ }^{1} \mathrm{H}$ NMR spectrum of 2 showed resonated signals at $\delta 6.52,5.5$ and 6.63 ppm attributed to $\mathrm{NH}$, styryl and $\mathrm{NH}_{2}$ protons, while that of 3 recorded a lack's of $\mathrm{NH}_{2}$ protons with the presence of $\mathrm{P}-\mathrm{H}$ proton at $\gamma 6.7 \mathrm{ppm}$ as a doublet resonating with a double constant of $6.35 \mathrm{~Hz} \cdot{ }^{13} \mathrm{C}$ NMR of compound 2 showed $\delta$ at 165.8, 167, 152 and $140 \mathrm{ppm}$ attributed to $\mathrm{C}=\mathrm{S}, \mathrm{C}=\mathrm{O}, \mathrm{C}=\mathrm{C}$ and $\mathrm{C}-\mathrm{Cl}$ carbons (Figure 5).

The IR spectrum of compound 4 recorded $\gamma$ at 1337 and $1120,1488 \mathrm{~cm}^{-1}$ for 


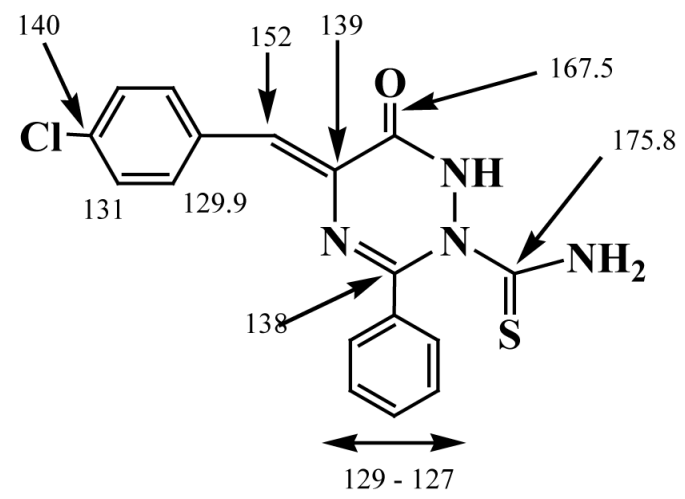

Figure $5 .{ }^{13} \mathrm{C}$ NMR spectrum of compound 2 .

$\mathrm{SO}_{2}$ and $\mathrm{CH}_{3}$ groups. Also, $\gamma$ at 3200,1180 and $720 \mathrm{~cm}^{-1}$ for $\mathrm{NH}, \mathrm{C}=\mathrm{S}$, and $\mathrm{C}-\mathrm{Cl}$ functional groups structure of 4 confirmed from ${ }^{1} \mathrm{H}$ NMR spectrum, were showed $\delta$ at 6.7, 6.52 and 2.55 ppm for P-H, NH and $\mathrm{CH}_{3}$ protons.

On the other hand, ${ }^{13} \mathrm{C}$ NMR spectrum of compound 5 exhibited different types of carbon atoms at $\delta 162,158(2 \mathrm{C}=\mathrm{O}), 145(\mathrm{C}-\mathrm{F}), 138\left(\mathrm{C}-\mathrm{SO}_{2}\right), 170(\mathrm{C}=\mathrm{S})$, $22(\mathrm{Me})$ and at $131-127$ (aromatic carbon). Mass fragmentation pattern of compound 5 recorded a molecular on the peak with $\mathrm{M}^{+2}$ due to isomeric, $\mathrm{F}$ and $\mathrm{Cl}$ atoms with a base peak at $\mathrm{m} / \mathrm{e} 139$ attribute to 4-chlorobenzylidene followed by 4-methylphenylsylfonyl radical at 155 (90\%) (Figure 6).

One aim of the present work is to synthesize of fluorine-substituted pyrazolo-1,2,4-triazino-1,2,4-triazine as wholly fused hetero-polycyclic nitrogen conjugated systems. Thus, the synthetic of these groups deduced to give new compounds 6-11 (Scheme 3). Thus, IR spectra of both the compounds 6, 9 \& 11 showed the new additional $\mathrm{C}=\mathrm{O}$ groups than the compound 2 , which confirmed their structures. ${ }^{13} \mathrm{C}$ NMR spectra of compounds $9,10 \& 11$ recorded the presence of $\mathrm{C}=\mathrm{S}$ and $\mathrm{C}-\mathrm{F}$ carbons at 180 and $145 \mathrm{ppm}$.

On the other hand, IR absorption spectra of all the compounds 8 \& 11 recorded a lack's of $\mathrm{NH}$ group, with the presence of two $\mathrm{C}=\mathrm{O}$ and $\mathrm{C}-\mathrm{F}$ groups at $\gamma$ 1710,1690 , and $1250 \mathrm{~cm}^{-1}$. Moreover, all the compounds 5-11 showed the $\gamma$ at $1200-1170 \mathrm{~cm}^{-1}$ for functional groups. Also, IR spectra of compound 9 \& 11 showed a three of true carbonyl groups $1740,1710 \& 1690 \mathrm{~cm}^{-1}$.

Also, ${ }^{1} \mathrm{H}$ NMR spectra of compounds 8 \& 11 showed a lack's of NH protons, which confirm their reactions. Mass spectrum of compound 11 recorded a splitting of the $\mathrm{COCF}_{3}$ fragment, followed by small ions and finally a base peak at 4-chlorobenzylidene radical at m/e 139 (Figure 7).

\section{Experimental}

Melting point determined with an Electrothermal Bibby Stuart Scientific melting point sample (U K). A Perkin Elmer Model RXI-FT IR system 55529 was used for recording IR spectra of the prepared compounds $\left(\mathrm{cm}^{-1}\right)$. A Bruker advance DPX 400 MHZ model uses TMS as internal standard was used for recording the 


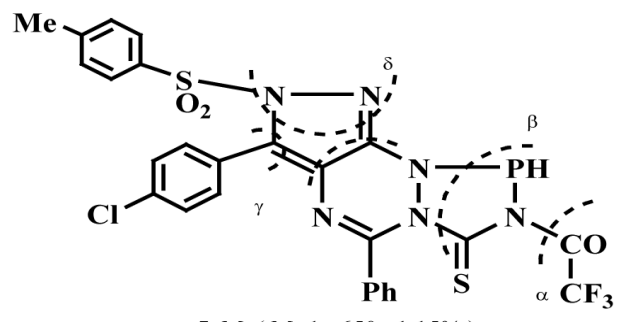

5. M. $(\mathrm{M}+1,650,1.15 \%)$

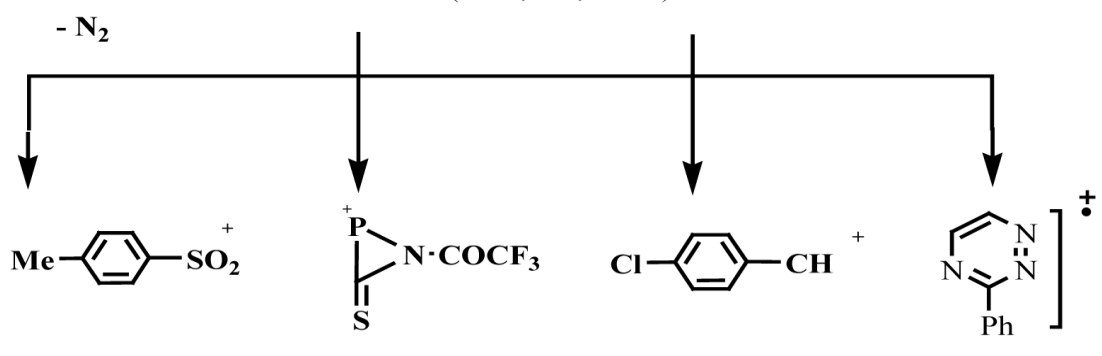

$155(90) \quad 155(90) \quad 139(100 \%)$

$157(8.15)$

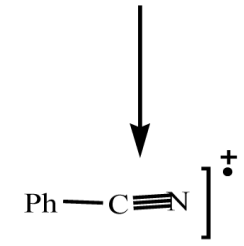

$103(15.0)$

Figure 6. Mass fragmentation pattern of compound 5.

6

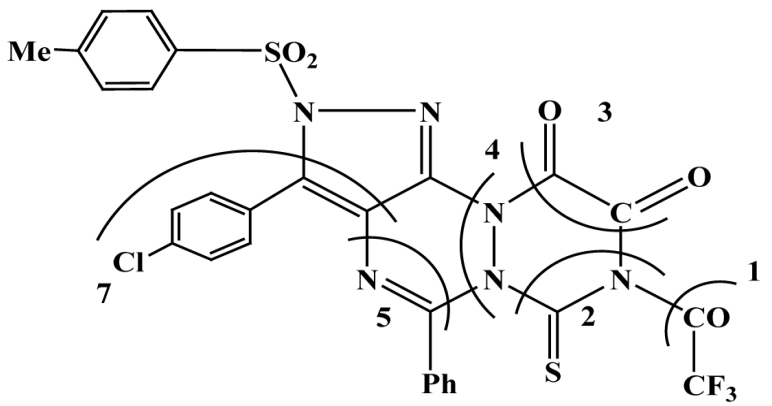

11, $\mathrm{M}^{+} 678(\mathrm{M}+2,77.8 \%)$

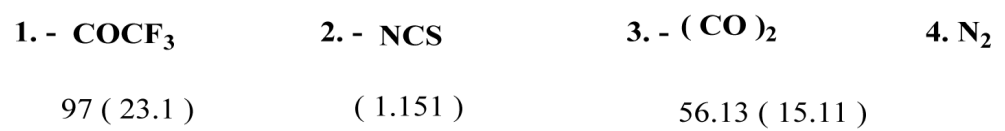

5. - Ph, - CN

$103(18.9)$
6.

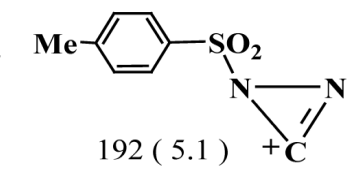

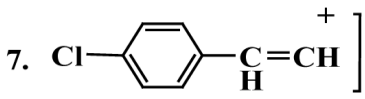

$138(100 \%)$

Figure 7. Mass fragmentation pattern of compound 11. 
${ }^{1} \mathrm{H}$ and ${ }^{13} \mathrm{C}$ NMR spectra of the compounds on deuterated DMSO- $\mathrm{d}_{6}$ (ppm). AGC-MS-GP 1000 Ex model used for recording the mass spectra of the compounds $(\mathrm{m} / \mathrm{z})$.

\section{1. (4'-Chlorobenzylidene)-2-Phenyl-0xazol-5-One (1)}

A mixture of 4-chlorobenaldehyde (0.01 mol), hippuric acid $(0.01 \mathrm{~mol})$, fused $\mathrm{NaOAc}(0.02 \mathrm{~mol})$, acetic anhydride $(0.02 \mathrm{~mol})$ and glacial acetic acid $(100 \mathrm{ml})$ refluxed for $2 \mathrm{~h}$, cooled then poured onto ice. The solid thus obtained filtered off and crystallized from EtOH to give 1; yield 80\%, m.p $140^{\circ} \mathrm{C}$. IR $(\gamma) \mathrm{cm}^{-1}: 1700$ $(\mathrm{C}=\mathrm{O}), 1610(\mathrm{C}=\mathrm{N}), 1596(\mathrm{C}=\mathrm{C}), 1070(\mathrm{C}-\mathrm{O}-\mathrm{C}), 850,830,810$ (phenyl CH), 710 (C-Cl) [8]. Anal. Calcd. for $\mathrm{C}_{16} \mathrm{H}_{10} \mathrm{NClO}_{2}$ (248), C, 67.60; H, 3.52; N, 4.92; Cl, 12.65\%. Found: C, $67.35 ; \mathrm{H}, 3.43 ; \mathrm{N}, 4.80 ; \mathrm{Cl}, 12.55 \%$.

\subsection{5-(4'-Chlorobenzylidene)-3-Phenyl-2-Aminothiocarbonyl- 1,2,4-Triazin-6-(1H)One (2)}

A mixture of $1(0.01 \mathrm{~mol})$ and thiosemicarbazide $(0.01 \mathrm{~mol})$ in acetic acid (50 $\mathrm{ml}$ ) heated under reflux for $3 \mathrm{~h}$. Then cold and poured onto ice. The solid produced filtered off and crystallized from acetic acid to give 2; yield 75\%, m.p $198^{\circ} \mathrm{C}-200^{\circ} \mathrm{C}$. IR $(\gamma) \mathrm{cm}^{-1}: 3250(\mathrm{NH}), 3159\left(\mathrm{NH}_{2}\right), 1710(\mathrm{C}=\mathrm{O}), 1620(\mathrm{C}=\mathrm{N})$, $1600(\mathrm{C}=\mathrm{C}), 1180(\mathrm{C}=\mathrm{S}), 900,850$ (aromatic $\mathrm{CH}), 708(\mathrm{C}-\mathrm{Cl}) .{ }^{1} \mathrm{H}$ NMR $\left(\right.$ DMSO-d $\left._{6}\right) \delta: 6.63\left(s, 2 \mathrm{H}, \mathrm{NH}_{2}\right), 6.88-7.77(\mathrm{~m}, 9 \mathrm{H}, \mathrm{Ar}-\mathrm{H}, \mathrm{H}$-olefinic), 10.8 $(s, 1 \mathrm{H}, \mathrm{NH}) \cdot{ }^{13} \mathrm{C}$ NMR DMSO $\left.-\mathrm{d}_{6}\right) \delta: 175.8(\mathrm{C}=\mathrm{S}), 167(\mathrm{C}=\mathrm{O}), 152(\mathrm{C}=\mathrm{C}), 140$ (C-Cl),130 - 120 (aromatic carbon). Anal. Calcd. For $\mathrm{C}_{17} \mathrm{H}_{13} \mathrm{~N}_{4} \mathrm{ClSO}$ (357), C, 57.14; H, 3.64; N, 15.68; Cl, 10.08\%. Found: C, 57.0; H, 3.45; N, 15.49; Cl, 9.89\%.

\subsection{7-(4'-Chlorobenzylidene)-9-Phenyl-2-Thioxo[5,1,2,4] Phospha[1,2,4]Trzole [1,2-a][1, 2,4]Triazin-6-One (3)}

A mixture of $2(0.01 \mathrm{~mol})$ and diethylphosphite $(0.01 \mathrm{~mol})$ in THF $(50 \mathrm{ml})$ refluxed for $2 \mathrm{~h}$, cooled. The obtained solid filtered off and crystallized from dioxan to give 3. Yield 70\%; m.p. $184^{\circ} \mathrm{C}-185^{\circ} \mathrm{C}$. IR $(\gamma) \mathrm{cm}^{-1}$ : $3300(\mathrm{NH}), 1710(\mathrm{C}=\mathrm{O})$, $1600(\mathrm{C}=\mathrm{C}), 1580(\mathrm{C}=\mathrm{N}), 1380$ (cyclic NCSN), $1190(\mathrm{C}=\mathrm{S}), 880,850$ (aromatic $\mathrm{CH}$ ), 700 (C-Cl). ${ }^{1} \mathrm{H}$ NMR (DMSO-d $\left.{ }_{6}\right) \delta: 6.7$ (d, P-H), $10.35(s, \mathrm{H}, \mathrm{NH}), 6.95$ 7.91( $m, 8 \mathrm{H}, \mathrm{Ar}-\mathrm{H} \& \mathrm{H}$-olefinic). Anal. Calcd. For $\mathrm{C}_{17} \mathrm{H}_{11} \mathrm{~N}_{4} \mathrm{ClSOP}$ (386), C, 62.17; H, 2.84; N, 14.50; Cl, 9.32; S, 8.29; P, 8.03\%. Found: C, 61.98; H, 2.75; N, $14.30 ; \mathrm{Cl}, 9.55 \%$.

\subsection{2-(4'-Tolylsulfonyl)-3-(4'-Chlorophenyl)-5-Phenyl-Pyrazolo $[4,3-e][1,2,4]$ Triazino $[1,2-a][5,1,2,4]$ Phosphatriazolo-7(8H) Thione (4)}

Equimolar amounts of 3 and 4-tolulene sulfonic acid hydrazide in ethanol (100 $\mathrm{ml}$ ), with a few drops of piperidine, refluxed for $8 \mathrm{~h}$, cooled then poured onto icedrops acetic. The solid produced filtered off and crystalized from dioxan to give 4, yield 65\%, m.p. $238^{\circ} \mathrm{C}-240^{\circ} \mathrm{C}$. IR $(\gamma) \mathrm{cm}^{-1}$ : $3200(\mathrm{NH}), 1600(\mathrm{C}=\mathrm{N}), 1488$ 
(deformation $\mathrm{CH}_{3}$ ), 1337 \& $1120\left(\mathrm{C}-\mathrm{SO}_{2}\right), 1188(\mathrm{C}=\mathrm{S})$, 900, 860, 810 (aromatic $\mathrm{CH}), 720(\mathrm{C}-\mathrm{Cl}) .{ }^{1} \mathrm{H}$ NMR (DMSO-d 6 ) $\delta: 6.7(s, 1 \mathrm{H}, \mathrm{P}-\mathrm{H}) 10.55(s, 1 \mathrm{H}, \mathrm{NH}), 7-$ $6\left(m, 13 \mathrm{H}\right.$, aromatic protons), 7.4, $7.2(d, d, 2 \mathrm{H}$ of $\mathrm{C}-\mathrm{Cl}), 1.05\left(s, 3 \mathrm{H}, \mathrm{CH}_{3}\right)$. Anal. Calcd. For $\mathrm{C}_{24} \mathrm{H}_{18} \mathrm{~N}_{6} \mathrm{ClS}_{2} \mathrm{O}_{2} \mathrm{P}$ (525): C, 54.85; H, 3.42; N, 10.66; Cl, 6.85; S, 12.19; P, 5.90\%. Found: C, 54.66; H, 3.25; N, 10.49; Cl, 6.55\%.

\subsection{2-(4'-Tollylsulfonyl)-3-(4'-Cholorophenyl)-5-Phenyl-8- Trifluoroacetyl)Pyrzolo[4,3-e][1,2,4]Triazino[1,2-a][5,1,2,4] Phosphatriazol-7-Thione (5)}

Equimolar mixture of 4 and trifluoro ethyl acetate in THF (50 ml) refluxed for 2 $\mathrm{h}$, cooled. The yielded solid filtered off and crystalized from dioxan to give $\mathbf{5}$. Yield 60\%, m.p. $218^{\circ} \mathrm{C}-220^{\circ} \mathrm{C}$, IR $(\gamma) \mathrm{cm}^{-1}: 1700(\mathrm{C}=\mathrm{O}), 1600(\mathrm{C}=\mathrm{N}), 1480$ (deformation $\mathrm{CH}_{3}$ ), 1335, $1130\left(\mathrm{C}-\mathrm{SO}_{2}\right), 1180$ (C=S), 1250 (C-F), 900, 880, 860, (aromatic CH), 705 (C-Cl), 650 (C-F). ${ }^{1} \mathrm{H}$ NMR (DMSO-d $\mathrm{d}_{6}$ ) $\delta: 6.75$ (s, P-H), 7.6, $7.4\left(d, d, 2 \mathrm{H}, 7.2-6.11\left(\mathrm{~m}, 13 \mathrm{H}\right.\right.$ aromatic protons), $0.95(s, 1 \mathrm{H}, \mathrm{CH} 3) .{ }^{13} \mathrm{C} \mathrm{NMR}$ $\left(\mathrm{DMSO}_{6} \mathrm{~d}_{6}\right) \delta: 170(\mathrm{C}=\mathrm{S}), 162(\mathrm{C}=\mathrm{O}), 145(\mathrm{C}-\mathrm{F}), 138\left(\mathrm{C}^{-S_{2}}\right), 131$ - 127 (aromatic carbons), $125(\mathrm{NCN}), 22\left(\mathrm{CH}_{3}\right) \cdot \mathrm{M} / \mathrm{S}($ Int.\% $)=650(\mathrm{M}+1,1.15 \%)$, 155(90.01), 139 (100\%). Anal. Calcd. For $\mathrm{C}_{26} \mathrm{H}_{17} \mathrm{~N}_{6} \mathrm{~F}_{3} \mathrm{ClS}_{2} \mathrm{O}_{3} \mathrm{P}$ (649): C, 48.07; $\mathrm{H}$, $2.61 ; \mathrm{N}, 12.94 ; \mathrm{F}, 8.78 ; \mathrm{Cl}, 5.54 ; \mathrm{S}, 9.86 ; \mathrm{P}, 4.77 \%$. Found: C, 47.88; H, 2.55; N, 12.90; F, 8.66; Cl, 5.40\%.

\subsection{6-(4'-Chlorobenzylidene)-4-Phenyl-2-Thioxo-1,2,4-Triazolo [1,2-a][1,2,4]Triazin-7,9 (1H)Dione (6)}

A mixture of $2(0.01 \mathrm{~mol})$ and diethyl carbonate $(0.01 \mathrm{~mol})$ in THF $(50 \mathrm{ml})$ refluxed $3 \mathrm{~h}$, cooled. The solid obtained filtered off and crystallized from dioxan to give 6.Yield $70 \%$, m.p. $190^{\circ} \mathrm{C}-192^{\circ} \mathrm{C}$, IR $(\gamma) \mathrm{cm}^{-1}: 3150(\mathrm{NH}), 1710,1690$ $(2 \mathrm{C}=\mathrm{O}), 1600(\mathrm{C}=\mathrm{C}), 1580(\mathrm{C}=\mathrm{N}), 1180(\mathrm{C}=\mathrm{S}), 880,850,810$ (aromatic $\mathrm{CH}), 706$ (C-Cl), ${ }^{1} \mathrm{H}$ NMR (DMSO-d $\left.\mathrm{d}_{6}\right) \delta: 10.35(s, 1 \mathrm{H}, \mathrm{NH}), 8.9(s, 1 \mathrm{H}$, olefinic proton), 7.8 - 6.2 (m, 8H, aromatic H). Anal. Calcd. For $\mathrm{C}_{18} \mathrm{H}_{11} \mathrm{~N}_{4} \mathrm{ClSO}_{2}$ (383): C, 56.39; $\mathrm{H}, 2.87 ; \mathrm{N}, 14.62 ; \mathrm{Cl}, 9.35 ; \mathrm{S}, 8.35 \%$. Found: $\mathrm{C}, 56.21 ; \mathrm{H}, 2.57 ; \mathrm{N}, 14.49 ; \mathrm{Cl}, 9.15$; S, $8.11 \%$.

\subsection{2-(4'-Tolylsulfonyl)-3-(4'-Chlorophenyl-5-Phenyl-7- Thioxo-Pyrazolo [4,3-e][1, 2,4]-Triazino[1,2-a][1,2,4] Triazol-9-(8H) One (7)}

Equimolar mixture of 6 and 4-tolune sulfonyl acid hydrazide in ethanol $(100 \mathrm{ml})$ with a few drops of piperidine, refluxed for $8 \mathrm{~h}$. Cooled then poured onto ice. The yielded solid filtered off and crytallized from EtOH to give 7. Yield $60 \%$, m.p. $258^{\circ} \mathrm{C}-260^{\circ} \mathrm{C}$, IR $(\gamma) \mathrm{cm}^{-1}: 3150(\mathrm{NH}), 1680(\mathrm{CONH}), 1590(\mathrm{C}=\mathrm{N}), 1480$ (deformation $\left.\mathrm{CH}_{3}\right), 1330\left(\mathrm{C}-\mathrm{SO}_{2}\right), 1188(\mathrm{C}=\mathrm{S}), 880,850,810$ (aromatic $\left.\mathrm{CH}\right), 700$ (C-Cl). ${ }^{1} \mathrm{H}$ NMR(DMSO-d $\left.\mathrm{d}_{6}\right) \delta: 10-11(s, 1 \mathrm{H}, \mathrm{NH}), 7.8,7.6(d, d, 2 \mathrm{H}, \mathrm{C}-\mathrm{Cl}), 7.4-$ $6.2(m, 3 \mathrm{H}$, aromatic $\mathrm{H})$. Anal. Calcd. For $\mathrm{C}_{25} \mathrm{H}_{17} \mathrm{~N}_{6} \mathrm{ClS}_{2} \mathrm{O}_{3}$ (549): C, 54.64; $\mathrm{H}$, 3.09; N, 15.30; Cl, 6.55; S, 11.65\%. Found: C, 54.51; H, 2.89; N, 15.11; Cl, 6.39; S, $11.51 \%$. 
4.8. 2-(4'-Tollylsulfonyl-3-(4'-Chlorophenyl)-5-Phenyl-7-Thioxo8-Trifluoroacetylpyrazolo $[4,3-e][1,2,4]$ Triazino $[1,2-a][1,2,4]$ Triazole (8)

A mixture of $7(0.01 \mathrm{~mol})$ and trifluoroethyl acetate $(0.01 \mathrm{~mol})$ in THF $(100 \mathrm{ml})$ refluxed for $2 \mathrm{~h}$, cooled. The solid obtained filtered off and crystallized from dioxan to give 8. Yield $66 \%$, m.p. $240^{\circ} \mathrm{C}-241^{\circ} \mathrm{C}$, IR $(\gamma) \mathrm{cm}^{-1}: 1710,1690$ $(2 \mathrm{C}=\mathrm{O}), 1480$ (deformation $\left.\mathrm{CH}_{3}\right), 1330\left(\mathrm{C}-\mathrm{SO}_{2}\right), 1250(\mathrm{C}-\mathrm{F}), 1189(\mathrm{C}=\mathrm{S}), 900$, 860, 810, (aromatic CH), 710 (C-Cl), 650 (C-F). ${ }^{1} \mathrm{H}$ NMR (DMSO-d $\left.\mathrm{d}_{6}\right) \delta: 7.8$ - 7.7 $(d, d, 2 \mathrm{H}, \mathrm{C}-\mathrm{Cl}), 7.4-7.2\left(d, d, 2 \mathrm{H}, \mathrm{C}-\mathrm{SO}_{2}\right), 7.0-6.0(m, 13 \mathrm{H}$, aromatic $\mathrm{H}) .{ }^{13} \mathrm{C}$ NMR (DMSO-d $\left.\mathrm{d}_{6}\right) \delta: 178(\mathrm{C}=\mathrm{S}), 168,162(2 \mathrm{C}=\mathrm{O}), 152(\mathrm{C}=\mathrm{N}), 145(\mathrm{C}-\mathrm{F})$, $149(\mathrm{NCN}), 131$ - 121 (aromatic carbons). Anal. Calcd. For $\mathrm{C}_{27} \mathrm{H}_{16} \mathrm{~N}_{6} \mathrm{~F}_{3} \mathrm{ClS}_{2} \mathrm{O}_{4}$ (645): C, 50.23; H, 2.48; N, 13.02; F, 8.83; Cl, 5.58; S, 9.92\%. Found: C, 50.11; H, $2.38 ; \mathrm{N}, 12.59 ; \mathrm{F}, 8.71 ; \mathrm{Cl}, 5.38 ; \mathrm{S}, 9.78 \%$.

\subsection{6-(4'-Chlorobenzylidene)-10-Phenyl-2-Thioxo-1,2,4- Triazino[1,2-a][1,2,4]Triazin-4,5,7(3H)Trione (9)}

A mixture of $2(0.01 \mathrm{~mol})$ and diethyl oxalate $(0.01 \mathrm{~mol})$ in THF $(50 \mathrm{ml})$ refluxed for $3 \mathrm{~h}$, coold. The yieled solid filtered off and crystallized from dioxan to give 9. Yield $72 \%$, m.p. $270^{\circ} \mathrm{C}-271^{\circ} \mathrm{C}$, IR $(\gamma) \mathrm{cm}^{-1}: 3200(\mathrm{NH}), 1740,1710,1690$ $(3 \mathrm{C}=\mathrm{O}), 1600(\mathrm{C}=\mathrm{C}), 1580(\mathrm{C}=\mathrm{N}), 1710(\mathrm{C}=\mathrm{S}), 880,810$ (aromatic $\mathrm{CH}), 700$ (C-Cl). ${ }^{1} \mathrm{H}$ NMR (DMSO-d $\left.\mathrm{d}_{6}\right) \delta: 11.10(s, 1 \mathrm{H}, \mathrm{NH}), 7.4,7.2(d, d, 2 \mathrm{H}, \mathrm{C}-\mathrm{Cl}), 7.0-$ $6.2\left(m, 9 \mathrm{H}\right.$, aromatic protons). Anal. Calcd. for: $\mathrm{C}_{19} \mathrm{H}_{11} \mathrm{~N}_{4} \mathrm{ClSO}_{3}$ (411): C, 55.47; H, 2.67; N, 13.62; Cl, 8.75; S, 7.7\%. Found: C, 55.47; H, 2.42; N, 13.51; Cl, 8.66; S, $7.55 \%$.

\subsection{2-(4'-Tollylsulfonyl-3-(4'-Chlorophenyl)-5-Phenyl-7- \\ Thioxopyrazolo[4,3-e][1,2,4]Triazino[1,2-a][1,2,4] \\ Triazin-9,10(8H)Dione (10)}

Equimolar mixture of 9 and 4-toluenesulfonic acid hydrazide in ethanol (50 ml) with few drops of piperidine refluxed for $8 \mathrm{~h}$, cooled then poured onto ice. The produced solid filtered off and crystallized from ethanol to give 10. Yield $68 \%$, m.p. $184^{\circ} \mathrm{C}-185^{\circ} \mathrm{C}$, IR $(\gamma) \mathrm{cm}^{-1}: 3150(\mathrm{NH}), 1700,1680(2 \mathrm{C}=\mathrm{O}), 1590(\mathrm{C}=\mathrm{N})$, 1488 (deformation $\left.\mathrm{CH}_{3}\right), 1335\left(\mathrm{C}-\mathrm{SO}_{2}\right), 1188(\mathrm{C}=\mathrm{S}), 890,860,810$ (aromatic $\mathrm{CH}), 710(\mathrm{C}-\mathrm{Cl}) .{ }^{1} \mathrm{H}$ NMR (DMSO-d $\left.{ }_{6}\right) \delta: 10.55(s, 1 \mathrm{H}, \mathrm{NH}), 7.6,7.4,7.2,7.0$ (each $d, d$, aromatic proton), $6.9-6.1$ ( $m, 13 \mathrm{H}$, aromatic protons). Anal. Calcd. for $\mathrm{C}_{26} \mathrm{H}_{17} \mathrm{~N}_{6} \mathrm{ClS}_{2} \mathrm{O}_{4}(577)$ : C, 54.07; H, 2.94; N, 14.55; Cl, 6.23 S, 11.09\% Found: C, $53.88 ; \mathrm{H}, 2.81 ; \mathrm{N}, 14.35 ; \mathrm{Cl}, 6.01 ; \mathrm{S}, 10.90 \%$.

\subsection{2-(4'-Tollylsulfonyl)-3-(4'-Chlorophenyl)-5-Phenyl-7- \\ Thioxo-8-Trifluoroacet-Ylpyrazolo[4,3-e][1,2,4] Triazino [1,2-a][1,2,4]Triazin-9,10-Dione (11)}

A mixture of $10(0.01 \mathrm{~mol})$ and trifluoroethyl acetate $(0.01 \mathrm{~mol})$ in THF $(50 \mathrm{ml})$ refluxed $2 \mathrm{~h}$, cooled. The solid produced filtered off and crystallized from dioxan to give 11 . Yield $55 \%$, m.p. $208^{\circ} \mathrm{C}-210^{\circ} \mathrm{C}$, IR $(\gamma) \mathrm{cm}^{-1}: 1720,1700,1690(3 \mathrm{C}=\mathrm{O})$, 
1600, $1580(\mathrm{C}=\mathrm{N}), 1488$ (deformation $\left.\mathrm{CH}_{3}\right), 1370\left(\mathrm{C}-\mathrm{SO}_{2}\right), 1250(\mathrm{C}-\mathrm{F}), 1185$ $(\mathrm{C}=\mathrm{S}), 860,840,810$ (aromatic CH), $710(\mathrm{C}-\mathrm{Cl}), 660(\mathrm{C}-\mathrm{F}) .{ }^{1} \mathrm{H}$ NMR $\left(\mathrm{DMSO}-\mathrm{d}_{6}\right.$ ) $\delta: 7.4,7.2,7.1$ - 7.0 (each $d, d, \mathrm{C}-\mathrm{Cl}, \mathrm{C}-\mathrm{SO}_{2}$, aromatic protons), $6.85-6.1(\mathrm{~m}$, $13 \mathrm{H}$, aromatic protons), $1.55(3 \mathrm{H} \mathrm{Me}) .{ }^{13} \mathrm{C} \mathrm{NMR}\left(\mathrm{DMSO}-\mathrm{d}_{6}\right) \delta: 180(\mathrm{C}=\mathrm{S}), 172$ $(\mathrm{C}=\mathrm{C}), 167(\mathrm{C}=\mathrm{O}), 164(\mathrm{C}=\mathrm{O}), 152(\mathrm{C}=\mathrm{N}), 145(\mathrm{C}-\mathrm{F}), 130$ - 120 (aromatic carbons). M/S (Int.\%): $\left.678 \mathrm{M}^{+2}, 77.8 \%\right), 192(5.11), 138(100), 103(18.9), 97(23.1)$, 56.13(15.11). Anal. Calcd. For $\mathrm{C}_{28} \mathrm{H}_{16} \mathrm{~N}_{6} \mathrm{~F}_{3} \mathrm{ClS}_{2} \mathrm{O}_{5}$ (673): C, 49.92; H, 2.37; N, 12.48; F, 8.46; Cl, 5.34; S, 9.50\% Found: C, 49.81; H, 2.11; N, 12.35; F, 8.31; Cl, $5.11 ; \mathrm{S}, 9.35 \%$.

\section{The Biological Activity}

Abdel-Rahman et al. [21]-[26], reported that most of the functionalized 1,2,4triazines substituted with fluorine atoms showed anticancer activity. In recent years, the synthesis fluorinated 1,2,4-triazinopyrazole and fused heterobicyclic system containing 1,2,4-triazine moiety have gained importance because of their biological activities [27] [28] [29]. Thus, novel fluorine substituted fused hetero-polycyclic nitrogen system containing pyrzolo1,2,4-triazine and 1,2,4-triazino1,2,4-triazine, have been synthesized in hope to improve their biological activities.

The synthesized compounds were evaluated for their antibacterial activity against the gram-positive bacterial strain. $S$. aureus and gram-negative bacterial strain. $S$. typhi, in addition, some fungi as $A$. niger according to the reported method [30] via DMF as a blank and Ciprofloxacin and Miconazole used as standard drugs. All the compounds exhibit antibacterial activity ranging from 50 to $150 \mathrm{mg} \mathrm{m} / \mathrm{ml}$. Also, the minimum inhibitory concentration (MIC) for the active compounds determined (Table 1 ).

Table 1 . The antimicrobial activity of the synthesized compounds $2-11$ by inhibition zones $(\mathrm{m} \mathrm{m})^{*}$.

\begin{tabular}{|c|c|c|c|}
\hline \multirow{2}{*}{$\begin{array}{c}\text { Sample } \\
\text { No. }\end{array}$} & \multicolumn{2}{|c|}{ Bacterial } & \multirow{2}{*}{$\begin{array}{l}\text { Fungi } \\
\text { A. niger }\end{array}$} \\
\hline & S. aurens & S. tyhi & \\
\hline 2 & 10.5 & 8.5 & 6.5 \\
\hline 3 & 12.5 & 10.5 & 11.5 \\
\hline 4 & 8.5 & 8.0 & 7.0 \\
\hline 5 & 12.5 & 11.5 & 12.0 \\
\hline 6 & 9.5 & 9.0 & 9.5 \\
\hline 7 & 8.5 & 8.0 & 10.0 \\
\hline 8 & 10.5 & 10.5 & 12.0 \\
\hline 9 & 8.0 & 7.0 & 8.0 \\
\hline 10 & 8.5 & 7.5 & 10.0 \\
\hline 11 & 10.5 & 11.5 & 17.5 \\
\hline DMF (Control) & 5 & 7 & 14 \\
\hline Stander & \multicolumn{2}{|c|}{5} & 5 \\
\hline
\end{tabular}

${ }^{*}$ Ciproflaxacin \& Miconazole (for bacteria) (for fungi) ${ }^{\star D M F}$ : used as control. 
From the results obtained we can be concluded that:

The presence of both the $\mathrm{COCF}_{3}$ and $\mathrm{P}$-atom led to the enhanced activity of compounds $3,5,8, \mathbf{1 1}$. The compounds containing 1,2,4-triazole-3-thione $(4,6)$ and 1,2,4-triazino-1,2,4-triazin-3-thione showed moderate activity.

The presence of aryl sulfonyl group with synthetic systems $(4-8 \& 10,11)$ also improves the antibacterial activity.

The synthetic compounds containing a phosphorus atom characterized as antifungal agents. It is clear that the biocidal effect's of the new synthesized systems towards $S$. aurens (bacteria) is more than the other tested bacteria and fungi, which it maybe the biodynamic formed between $S$. aurens and synthetic systems are considered a strong state. Also, the effects of DMF as control is highly on S. aurens than other bacteria and moderate effect's on the tested fungi.

Finally, the compounds 3, 5, 8, \& 11 exhibited a high antimicrobial activity which may be due to the presence a type of bio-dynamic systems, via the interaction between the microbial and the fluorinated compound synthesized.

\section{Conclusion}

New fluorinated fused hetero-polycyclic nitrogen systems containing pyrazolo1,2,4-triazine moiety and sulfonyl groups have been synthesized and evaluated as antimicrobial agents. The results obtained showed that presence of trifluoro acetyl, a phosphorus atom, and sulfonyl groups enhanced the activities. The higher activity of compound 5 can be attributed to the presence of phosphorus atom as donation and back-donation of the electron, with additive properties of $\mathrm{CF}_{3}$ groups also of sulfonyl group at the terminal of fused heteroconjugation systems formed.

\section{References}

[1] Abdel-Rahman, R.M. and Saad, H.A. (2016) Synthesis and Chemical Behavior of 1,2,4-Triazine Derivatives Bearing Phosphorus Amides as Donor-Acceptors: A Review. Current Organic Synthesis, 13, 408-425. https://doi.org/10.2174/1570179412666150905001956

[2] Garcia-Hemandez, Z., Floress-Parra, A., Grery, J.M., Ramos-Organilo, A. and Contreras, R. (2006) 2-Amino Benzothiazole Phosphorus Amides: Molecular and Supramolecular Structures, hydrogen Bonds and Sulfur Donor-Acceptor Interaction. Polyhedron, 25, 1662-1672. https://doi.org/10.1016/j.poly.2005.11.018

[3] Ali, T.E., Abdel-Rahman, R.M., Hanafy, F. and El-Edfawy, S.M. (2008) Synthesis and Molluscicidal Activity of Phosphorus Containing Heterocyclic Compounds Derived from 5,6-Bis(4-bromophenyl)-3-hydrazino-1,2,4-triazine. Phosphorus, Sulfur, and Silicon and the Related Elements, 183, 2565-2577. https://doi.org/10.1080/10426500801967864

[4] Al-Romaizan, A.N., Makki, M.S.T. and Abdel-Rahman, R.M. (2014) Synthesis of New Fluorine/Phosphorus Substituted 6-(2'-Aminophenyl)-3-thioxo-1,2,4-triazin$5(2 \mathrm{H}, 4 \mathrm{H})$ one and their related Alkylated Systems as Molluscicidal Agents as against the Snails Responsible for Bilharzias Diseases. International Journal of Organic Chemistry, 4, 154-168. https://doi.org/10.4236/ijoc.2014.42017

[5] Abdel-Rahman, R.M., Makki, M.S.T. and Al-Romizan, A.N. (2014) Synthesis of 
Novel Fluorine Substituted Isolated and Fused Heterobicyclic Nitrogen Systems Bearing 6-(2'-Phosphorylanilido)-1,2,4-Triazin-5-one Moiety as Potential Inhibitors towards WIV-1 Activity. International Journal of Organic Chemistry, 4, 247-268. https://doi.org/10.4236/ijoc.2014.44028

[6] Zein, M.A. and El-Shenawy, A.I. (2016) An Efficient Synthesis of 1,2,4-Triazine-6One Derivatives and Their In Vitro Anticancer Activity. International Journal of Pharmacology, 12, 188-194. https://doi.org/10.3923/ijp.2016.188.194

[7] Al-Hussaini, A.S., El-Sayed, E.H. and Radwan, E.M. (2015) Synthesis of 2,3,5-Trisubstituted 1,2,4-Triazine-6-Ones as Potential Antitumor. Der Pharma Chemica, 7, 214-225.

http://www.derpharmachemica.com/archive/dpc-volume-7-issue-11-year-2015.html

[8] El-Hady, H.A. and El-Sakka, S.E. (2014) Synthesis and Antitumor Activity of Some 1,2,4-Triazine Derivatives. International Journal of Innovative Research in Science Engineering and Technology, 3, 10854-10860.

[9] Abubshait, S.A. and Abubshait, H.A. (2012) Biological Activity and Mass Spectra Investigation of Synthesized 1,2,4-Triazine Derivatives. Journal of Chemical \& Pharmaceutical Research, 4, 568-579.

http://www.joccpr.com/articles/biological-activity-and-mass-spectra-investigationof-synthesized-1-2-4triazine-derivatives.pdf

[10] Abdullatif, M.H. (2016) Synthesis of Some Novel Compounds of Saccharinyl Acetic Acid Containing Nucleus and Evaluation of Their Biological Activities as Antimicrobial. Oriental Journal of Chemistry, 32, 567-574.

[11] Shaikh, B.M., Chobe, S.S., Konda, S.G., Khandara, N.T., Chavan, S.A. and Dawane, B.S. (2010) An Efficient Synthesis and In Vitro Antimicrobial Activity of 1,2,4-Triazin-6-(5H)one Derivatives. Der Chemica Sinica, 1, 86-91.

[12] Dawane, B.S., Kadam, S.N. and Shaikh, B.M. (2010) An Efficient Synthesis of 1,2,4-Triazine Derivatives and Their In Vitro Antimicrobial Activity. Der Pharmacia Letter, 2, 126-131.

[13] Khalifa, M.M.A. (2006) Synthesis and Antimicrobial Evaluation of Novel 1,2,4-Triazine Derivatives. Oriental Journal of Chemistry, 24, 825-830.

[14] Mulwad, V.V. and Satwe, D.S. (2006) Synthesis of Biological Active 4-[1-(2H)[1]-4Hydroxy-2-oxo-benzopyran-3-yl)methylidene]-2-phenyl-4H-oxazol-5-ones and 1,2,4Triazine-6-one and Its Derivatives. Indian Journal of Chemistry, 45, 1210-1215.

[15] Hin, N., Duvall, B., Ferraris, D., Alt, J., Thomas, A.G., Rais, R., Raja, C., Wu, Y., Wozniak, K.M., Slusher, B.S. and Tsukamoto, T. (2015) 6-Hydroxy-1,2,4-Triazine-3,5- $(2 \mathrm{H}, 4 \mathrm{H})$-dione Derivatives as Novel D-Amino Acid Oxidase Inhibitors. Journal of Medicinal Chemistry, 58, 7258-7272. https://doi.org/10.1021/acs.jmedchem.5b00482

[16] El-Abadelah, M.M., Abushamleh, A.S., Mossmer, C.M. and Voelter, W. (2002) Transitione Metal Complexes of Derivatized Chiral Dihydro-1,2,4-triazin-6-ones, Part IV. Synthesis and Structural Characterization of a Copper(II) Complexes L-3-acetyl-5-benzyl-1-phenyl-4,5-dihydro-1,2,4-triazin-6-one Oxime. Zeitschrift for Naturforschung B, 57, 547-551.

[17] Abdel-Rahman, R.M. and Bakhotmah, D.A. (2016) Synthesis and Chemistry of Bioactive Pyrazolyl-1,2,4-Triazines. Mini Review in Organic Chemistry, 13, 62-77.

[18] Makki, M.S.T., Abdel-Rahman, R.M. and Khan, K.A. (2014) Fluorine Substituted 1,2,4-Triazinones as Potential Anti-HIV-1 and CDK2 Inhibitor. Journal of Chemistry, 2014, Article ID 430573. https://doi.org/10.1155/2014/430573

[19] Abdel-Rahman, R.M. (1999) Chemistry of Uncondensed 1,2,4-Triazines, Part III. 
Synthesis and Chemistry of Fluorine Containing Bioactive 1,2,4-Triazines Overview. Pharmazie, 54, 791-863.

[20] Abdel-Rahman, R.M., Bakhotmah, D.A. and Fakhorji, M. (2017) Synthesis and Chemical Reactivity of Some New Fluorine Substituted Pyrazolopyrimidine Derivatives and Their Effect on Cellobiase Activity Produced by Fungi. World Journal of Organic Chemistry, 5, 1-5.

[21] Abdel-Rahman, R.M. (1992) Synthesis of Some New Fluorine Bearing Trisubstituted 3-Thioxo-1,2,4-triazin-5-ones as Potential Anticancer Agents. Farmaco, 47, 319.

[22] Abdel-Rahman, R.M., Seada, M., Fawzy, M. and El-Baz, I. (1993) Synthesis of Some New Thioethers Bearing a 1,2,4-Triazine-3-hydrazones and Assays for Their Anticancer and Anti-Human Immune Virus Activities. Farmaco, 48, 391-406.

[23] Abdel-Rahman, R.M., Morsy, J., Hanafy, F. and Amine, H.A. (1999) Synthesis of Heterobicyclic Nitrogen Systems Bearing the 1,2,4-Triazine Moiety as Anti-HIV and Anticancer Drugs-I. Pharmazie, 54, 347-351.

[24] Abdel-Rahman, R.M., Morsy, J., El-Edfawy, S. and Amine, H.A. (1999) Synthesis of Some New Heterobicyclic Nitrogen Systems Bearing the 1,2,4-Triazine Moiety as Anti-HIV and Anticancer Drugs II. Pharmazie, 54, 667-671.

[25] El-Gendy, Z., Morsy, J., Allimony, H., Abdel-Monem, W. and Abdel-Rahman, R.M. (2001) Synthesis of Some New Heterobicyclic Nitrogen Systems Bearing 1,2,4-Triazine Moiety as Anti-HIV and Anticancer Drug-III. Pharmazie, 56, 376.

[26] El-Gendy, Z., Morsy, J., Allimony, H., Abdel-Monem, W. and Abdel-Rahman, R.M. (2003) Synthesis of Some New Heterobicyclic Nitrogen Systems Bearing 1,2,4-Triazine Moiety as Anticancer Drugs: Part IV. Phosphorus, Sulfur, and Silicon and the Related Elements, 178, 2055-2071. https://doi.org/10.1080/10426500390228738

[27] Bakhotmah, D.A. and Abdel-Rahman, R.M. (2017) Synthesis and Structural Determination of Novel Fluorinated Steroidal Spiro(pyrazolo[4,3-e][1,2,4]triazin-3'-yl) Derivatives as Affecting Enzymatic Agents. Letter in Organic Chemistry, 14, 134-140. https://doi.org/10.2174/1570178614666161230143228

[28] Abdel-Rahman, R.M., Angawi, R.F. and Al-Mehmady, A.R. (2017) Synthesis and Biological Evaluation of Fluorine-Substituted pyrazolo[4,3-e][1,2,4]triazines as $\mathrm{Pu}$ rine Analogues. Journal of Saudi Chemical Society, 21, 495-503. https://doi.org/10.1016/j.jscs.2016.11.004

[29] Aqlan, F.M., Makki, M.S.T. and Abdel-Rahman, R.M. (2016) Synthesis, Spectroscopic Studies of Fluorinated Pyrimido-1,2,4-Triazines: Protective Effect against Some Plant Pathogenic Fungi. Journal of Heterocyclic Chemistry, 35, 1310-1317. https://doi.org/10.1002/jhet.2386

[30] Muanza, D.N., Kim, B.W., Euler, K.L. and Dan Williams, L. (1994) Antibacterial and Antifungal Activities of Nine Medicinal Plants from Zaire. International Journal Pharmacognosy, 32, 337-345. https://doi.org/10.3109/13880209409083012 